Abstract OWE-03 Table 1

\begin{tabular}{|c|c|c|c|}
\hline Characteristic & $\begin{array}{l}\text { Combined clinical-biochemical remission } \\
\qquad(\mathrm{n}=24)\end{array}$ & $\begin{array}{l}\text { Not in combined clinical-biochemical remission } \\
\qquad(\mathrm{n}=22)\end{array}$ & $p$ \\
\hline Gender, male:female & $18: 6$ & 9:13 & 0.035 \\
\hline Median age (years) & 33 & 36 & 0.75 \\
\hline Concomitant immunomodulator & $18(75 \%)$ & $17(77 \%)$ & $>0.99$ \\
\hline Prior anti-TNF experience & $0(0 \%)$ & $2(9 \%)$ & 0.22 \\
\hline Maintenance dose, $50 \mathrm{mg}: 100 \mathrm{mg}$ & $12: 12$ & $10: 12$ & 0.78 \\
\hline Median body mass index & 23.5 & 25.0 & 0.37 \\
\hline \multicolumn{4}{|l|}{ Disease activity } \\
\hline Median SCCAI & 0 & 5 & $<0.0001$ \\
\hline Median PRO2 & 0 & 2.5 & $<0.0001$ \\
\hline Median Calprotectin (ug/g) & 17 & 419 & $<0.0001$ \\
\hline Median CRP (mg/L) & 1 & 1 & 0.17 \\
\hline Median Albumin (g/L) & 47 & 46 & 0.042 \\
\hline \multicolumn{4}{|l|}{ Quality of life } \\
\hline Median IBD-Control-8 & 16 & 6 & $<0.0001$ \\
\hline Median IBD-Control-Visual Analogue & 92 & 49 & $<0.0001$ \\
\hline \multicolumn{4}{|l|}{ Scale } \\
\hline \multicolumn{4}{|l|}{ Golimumab Measurement } \\
\hline Median Serum Golimumab & 3.0 & 2.0 & 0.031 \\
\hline Concentration (ug/ml) & & & \\
\hline
\end{tabular}

threshold to achieve combined remission (sensitivity 0.75 , specificity 0.59, AUC 0.69). No AGA were detected.

Conclusions The GO-LEVEL maintenance cohort offers further evidence of the exposure-response relationship with golimumab, particularly when using a combined definition of remission that includes an objective marker of disease activity (FC). Clinicians may consider using therapeutic drug monitoring to optimise golimumab dosing aiming to achieve our suggested SGC therapeutic threshold of $2.1 \mathrm{ug} / \mathrm{ml}$.

\section{OWE-04 A CAPTURE-RECAPTURE STUDY OF ALL-AGE IBD POINT PREVALENCE IN SCOTLAND}

${ }^{1}$ Gareth-Rhys Jones*, 'Mathew Lyons, 'Cathy Bisset, ${ }^{1}$ Nikolas Plevris, ${ }^{1}$ Philip Jenkinson, ${ }^{1}$ James Fulforth, ${ }^{1}$ Kate Kirkwood, ${ }^{1}$ Shaun Chuah, 'Sarah Minnis, 'Sarah-Louise Gillespie, ${ }^{1}$ William Brindle, ${ }^{2}$ Christopher Burgess, ${ }^{2}$ Paul Henderson, ${ }^{1}$ Charlie Lees, ${ }^{2}$ David Wilson. ${ }^{1}$ Western General Hospital, NHS Lothian, Edinburgh, UK; ${ }^{2}$ Royal Hospital for Sick Children, NHS Lothian, Edinburgh, UK

10.1136/gutjnl-2019-BSGAbstracts. 125
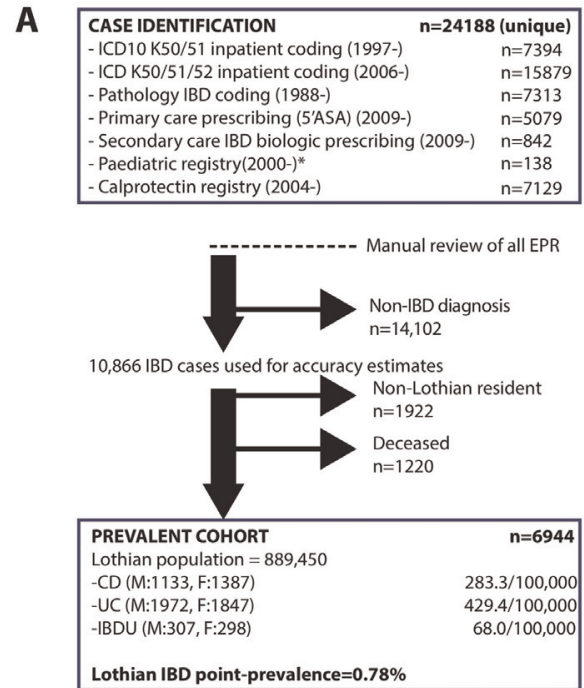

Figure 1 The Lothian IBD Registry (LIBDR)

(A) Flow chart of LIBDR data sources and prevalent cohort details, (B) IBD subtype point prevalence on 01/08/18 with age /sex breakdown and shaded proportion representing prevalent cases in active follow up

* A prospective paediatric registry has recorded prevelant IBD cases from 2000- (point-prevalence case number on 01/8/18 displayed).
B

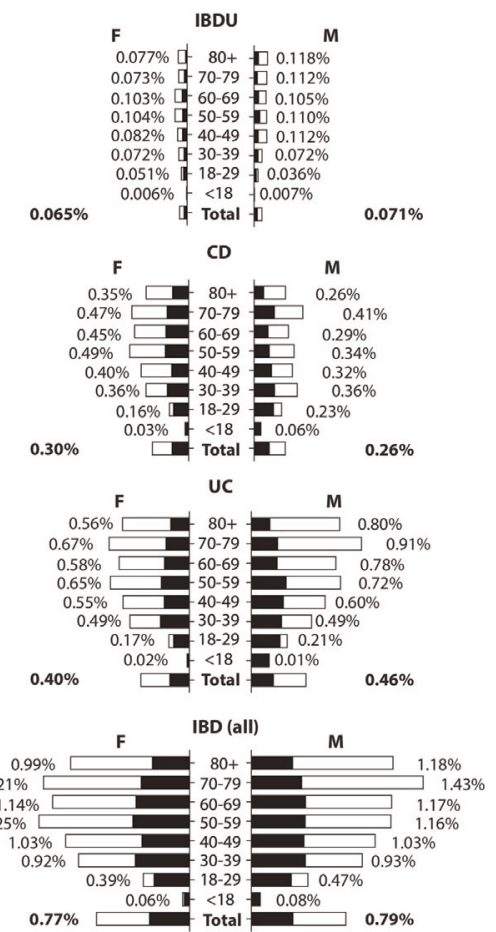


Background A recent systematic review reports an overall prevalence for IBD of $0.3 \%$ in Western countries ${ }^{1}$ but there are no UK estimates since 2003. We aimed to report current all-age prevalence, healthcare usage and forecast 2028 caseload, for IBD in Lothian.

Methods We conducted an extensive capture-recapture search strategy, manually confirming all diagnoses through electronic patient record (EPR) review, to provide point prevalence estimates for Lothian, Scotland (Population 889,450). Patients were identified from inpatient IBD codes (K50/51/52), IBD pathology codes, IBD biologic prescriptions, primary care mesalazine prescriptions, an existing calprotectin database and a paediatric IBD registry to identify 'possible' IBD cases to 31/08/18.

Eight IBD physicians then manually screened the EPR for all possible cases to identify true cases as per Lennard-Jones/ Porto criteria. Prevalence was calculated using postcode, date of diagnosis/death and population estimates from National Records Scotland. We then assessed our IBD cohort for attendance at secondary care, IBD-related admission, IBD-drug usage and projected 2028 Lothian IBD prevalence using autoregressive integrated moving average (ARIMA) modelling.

Results 24,188 possible IBD case records were manually reviewed to exclude non-IBD cases, leaving 10,866 true positives (figure $1 \mathrm{~A}$ ). The point prevalence of IBD in Lothian on $31 / 8 / 18$ was $0.78 \%$ (figure $1 \mathrm{~A}$ ).

$43.8 \%$ of prevalent cases attended out-patient clinic over the preceding 3 years which was inversely correlated with increasing age (Mean age $46.7 \pm 0.3$ in follow up versus 54.9 \pm 0.3 not in follow up, $\mathrm{p}<0.0001$ )(figure $1 \mathrm{~B}$ ). Follow-up was significantly associated with IBD-related admission (OR 5.7 $\{4.6-6.9\}, \mathrm{p}<0.0001)$ and IBD-related admission duration was positively correlated with increasing age $\left(\mathrm{R}^{2}=0.02\right.$, slope $=0.12$ $\{0.09-0.15\}, \mathrm{p}<0.0001)$.

ARIMA modelling projects a point prevalence on $01 / 08 / 28$ of $0.99 \%(0.94-1.04 \%)$ affecting $1.6 \%(1.5-1.7 \%)$ of the $>50$ s who will account for $59 \%$ of prevalent IBD.

Conclusions The prevalence of IBD in the UK is $0.78 \%$ (1 in 125 individuals). This is much higher than previously reported, and will continue to rise due to compound prevalence, reaching $>1.0 \%$ by the end of 2028 .

\section{REFERENCE}

1. Siew $\mathrm{Ng}$, et al. Worldwide incidence and prevalence of inflammatory bowel disease in the 21st century: a systematic review of population-based studies. Lancet 2018;390:pp2769-2778.

\section{OWE-05 INTERIM LONG-TERM SAFETY/EFFICACY OF RISANKIZUMAB TREATMENT IN CROHN'S DISEASE PATIENTS FROM THE OPEN-LABEL EXTENSION STUDY}

${ }^{1} \mathrm{M}$ Ferrante*, ${ }^{2} \mathrm{~J}$ Panes, ${ }^{3} \mathrm{~F}$ Baert, ${ }^{4} \mathrm{E}$ Louis, ${ }^{5} \mathrm{~A}$ Kaser, ${ }^{6} \mathrm{D}$ Gustafson, ${ }^{7} \mathrm{D}$ Hall, ${ }^{7}$ WO Böcher, ${ }^{7}$ I Herichova, ${ }^{6} \mathrm{X}$ Liao, ${ }^{6} \mathrm{~K}$ Wallace, ${ }^{8} \mathrm{~J}$ Kalabic, G D'Haens. ${ }^{7}$ University Hospitals Leuven, Leuven, Belgium; ${ }^{2}$ Hospital Clinic Barcelona, IDIBAPS, CIBERehd, Barcelona, Spain; ${ }^{3} A Z$ Delta Roeselare-Menen, Roeselare, Belgium; ${ }^{4}$ University of Liege and CHU, Liege, Belgium; ${ }^{5}$ University of Cambridge, Cambridge, UK; ${ }^{6}$ AbbVie Inc., North Chicago, USA; ${ }^{7}$ Boehringer Ingelheim International GmbH, Ingelheim am Rhein, Germany; ${ }^{8}$ AbbVie Deutschland GmbH and Co. KG, Ludwigshafen, Germany; ${ }^{9}$ Academic Medical Center, Amsterdam, Netherlands

\subsection{6/gutjnl-2019-BSGAbstracts. 126}

Introduction Adults with moderate-to-severe Crohn's disease (CD) who responded to risankizumab (RZB) in the phase 2 induction and maintenance study (Feagan, 2017) could enroll in an open-label extension (OLE) study. Interim efficacy and safety of RZB maintenance treatment from the OLE, up to 2 years, are reported.

Methods Patients (pts) achieving clinical response (decrease from baseline $[\mathrm{BL}]$ in $\mathrm{CD}$ Activity Index $[\mathrm{CDAI}] \geq 100$ ) without remission (CDAI <150) after wk 26 or clinical response and/or remission after wk 52 of the preceding study received $180 \mathrm{mg}$ s.c. RZB every 8 wks for up to 216 weeks. Pts losing clinical response or remission after completing the preceding study were re-induced with $600 \mathrm{mg}$ i.v. RZB at wk $0,4,8$. Pts received subsequent $180 \mathrm{mg}$ s.c. RZB maintenance treatment only if they achieved response or remission following re-induction treatment. Ileocolonoscopy was performed yearly. Efficacy data (clinical remission and endoscopic remission [CD Endoscopic Index of Severity (CDEIS) $\leq 4$ or CDEIS $\leq 2$ for pts with initial isolated ileitis]) are reported up to wk 48 . Non-responder imputation (NRI) was used for missing data.

Results A total of 65 pts were enrolled (including 4 who were re-induced). Mean (standard deviation) exposure to RZB was 657.2 (190.73) days. At the data cut-off, 14 (21.5\%) pts have discontinued the study. Up to wk 48, clinical remission rates were sustained and the proportion of pts with endoscopic remission increased from BL (table 1). Adverse events (AEs) were reported for $58(89.2 \%)$ pts; $18(27.7 \%)$ pts had serious AEs. AEs occurring in $>10 \%$ of pts were nasopharyngitis (26.2\%), fatigue (16.9\%), arthralgia and worsening CD $(15.4 \%$ each). Four serious infections in 5 pts were perianal abscess $(n=1)$, Campylobacter $(n=1)$, viral gastroenteritis $(n=2)$, and peritonitis $(n=2)$. No events of tuberculosis, malignancies or deaths occurred.

Conclusions In this interim analysis, clinical remission and endoscopic remission were sustained in CD pts receiving longterm RZB treatment. The safety profile of RZB was consistent with previously published data (Feagan, 2017).

Abstract OWE-05 Table 1 Pts in the OLE achieving clinical remission and endoscopic remission by visit

\begin{tabular}{lll}
\hline & ${\text { Clinical remission } n(\%)^{\mathrm{a}}}$ & Endoscopic remission $^{\mathrm{b}} \mathrm{n}(\%)^{\mathrm{a}}$ \\
\hline Wk $0^{\mathrm{c}}$ & $48(73.8)$ & $27(41.5)$ \\
Wk 8 & $47(72.3)$ & \\
Wk 16 & $46(70.8)$ & \\
Wk 32 & $48(73.8)$ & $35(53.8)$ \\
Wk 48 & $45(69.2)$ &
\end{tabular}

All pts had $\geq 48$ wks observation at the data cut-off ${ }^{\mathrm{a}} \mathrm{N}=65^{\mathrm{b}}$ Data from central reading ${ }^{\mathrm{C} V} \mathrm{~V}$ sits in OLE

\section{REFERENCES}

1. Feagan BG, et al. Lancet 2017,29;389(10080):1699-1709.

2. Feagan BG, et al. Gastroenterology 2017;152(5):S1310.

\section{OWE-37 CD4 T-CELL HLA-U PSEUDOGENE AT BASELINE PREDICTS CLINICAL REMISSION TO ANTI-TNF AGENTS IN ULCERATIVE COLITIS}

${ }^{1}$ Sreedhar Subramanian*, ${ }^{3}$ Lucille Rainbow, ${ }^{3}$ Matthew Gemmell, ${ }^{3}$ Yongxiang Fang, ${ }^{3}$ Sam Haldenby, ${ }^{3}$ Rachael Hough, ${ }^{2}$ Chris Probert. ${ }^{1}$ Department of Gastroenterology, Royal Liverpool University Hospital, Liverpool, UK; ${ }^{2}$ Institute of translational medicine, University of Liverpool, Liverpool L69 3BX, UK; ${ }^{3}$ Centre for genomics research, University of Liverpool, Liverpool L69 3BX, UK

\subsection{6/gutjnl-2019-BSGAbstracts. 127}

Introduction Anti-tumour necrosis factor (TNF) agents are used to treat UC but response is variable. Apart from 